\title{
Correlation between total pressure losses of highly loaded annular diffusers and integral stage design parameters
}

\section{Original article}

Article history:

Accepted: 20 June 2018

Published: 27 August 2018

Check for updates

*Correspondence:

DM: mimicatfd.uni-hannover.de

\section{Peer review:}

Single blind

\section{Copyright:}

(c) 2018 Mimic et al. (-) This is an open access article distributed under the Creative Commons Attribution License (CC-BY 4.0), which permits unrestricted use, distribution, and reproduction in any medium, provided the original work is properly cited and its authors credited.

\section{Keywords:}

diffuser; design correlation; total pressure; turbine; combined design; secondary flow

\section{Citation:}

Mimic D., Jätz C., and Herbst F. (2018). Correlation between total pressure losses of highly loaded annular diffusers and integral stage design parameters. Journal of the Global Power and Propulsion Society. 2: 388-401.

https://doi.org/10.22261/JGPPS.19AB30

\author{
Dajan Mimic (ID) ${ }^{1, *}$, Christoph Jätz ${ }^{2}$, Florian Herbst ${ }^{1}$ \\ ${ }^{1} J$ unior Research Group Multiphysics of Turbulent Flows, Institute of Turbomachinery and Fluid \\ Dynamics, Leibniz Universität Hannover, Appelstrasse 9, 30167 Hanover, Germany \\ ${ }^{2}$ Institute of Turbomachinery and Fluid Dynamics, Leibniz Universität Hannover, Appelstrasse 9, \\ 30167 Hanover, Germany
}

\section{Abstract}

Diffusers convert kinetic flow energy into a rise in static pressure. This pressure recovery is the primary aerodynamic design objective for exhaust gas diffusers in power-generating steam and gas turbines. The total pressure loss is an equally important diffuser design parameter. It is strongly linked to the pressure recovery and the residual kinetic energy of the diffuser outlet flow. A reduction benefits the overall thermodynamic cycle, which requires the adjacent components of a diffuser to be included in the design process.

This paper focuses on the total pressure losses in the boundary layer of a highly loaded annular diffuser. Due to its large opening angle the diffuser is susceptible to flow separation under uniform inlet conditions, which is a major source for total pressure losses. However, the unsteady tip leakage vortices of the upstream rotor, which are a source of losses, stabilise the boundary layer and prevent separation. Experiments and unsteady numerical simulation conducted show that the total pressure loss reduction caused by the delayed boundary layer separation exceed the vortex-induced losses by far. This flow interaction between the rotor and diffuser consequently decreases the overall total pressure losses.

The intensity of the tip leakage vortex is linked to three rotor design parameters, namely work coefficient, flow coefficient and reduced bladepassing frequency. Based on these parameters, we propose a semi-empiric correlation to predict and evaluate the change in total pressure losses with regards to design operating conditions.

\section{Introduction}

Diffusers make an essential contribution to increase the efficiency of power-generating steam and gas turbines. They harvest the otherwise wasted kinetic energy of the turbine exhaust flow and convert it into pressure-volume work, i.e., an increase in static pressure. For a given static outlet pressure a diffuser enables a higher turbine pressure ratio, resulting in an increase in power output. As the heat input of the thermodynamic cycle remains constant the thermal efficiency increases as well. The primary aerodynamic design goal of an exhaust diffuser is to maximise the kinetic energy conversion of the turbine 
outlet flow. The effectiveness of this process can be evaluated using the dimensionless pressure recovery $c_{p}$ defined as

$$
c_{p}=\frac{p_{\text {out }}-p_{\text {in }}}{p_{\text {tot,in }}-p_{\text {in }}}
$$

Assuming an ideal flow, it can be shown that an infinite area ratio AR of outlet to inlet area is required to convert all kinetic energy of the flow into static pressure. For such an ideal diffuser, the pressure recovery coefficient is defined as

$$
c_{p, \text { ideal }}=1-\frac{\left(1 / \mathrm{AR}^{2}\right)+\tan ^{2} \alpha\left(\boldsymbol{r}_{\text {in }} / \boldsymbol{r}_{\text {out }}\right)^{2}}{1+\tan ^{2} \alpha}
$$

where $\alpha=\tan ^{-1}\left(c_{\vartheta} / c_{m}\right)$ is the swirl angle at diffuser inlet with $\boldsymbol{r}_{\text {in }}$ and $\boldsymbol{r}_{\text {out }}$ as the Euler radii at inlet and outlet, respectively. It represents the theoretical limit for the pressure recovery of a certain diffuser geometry for a given swirl angle. The effectiveness of the diffuser $\epsilon$ is defined as

$$
\epsilon=\frac{c_{p}}{c_{p, \text { ideal }}}
$$

The outlet flow of a real exhaust diffuser with a finite area ratio has residual kinetic energy. This kinetic energy is characterised by the kinetic energy coefficient $\xi$

$$
\xi=\frac{p_{\text {tot,out }}-p_{\text {out }}}{p_{\text {tot,in }}-p_{\text {in }}}
$$

In contrast to an ideal inviscid flow, real thermodynamic processes are not completely reversible and thus entropy is created. This creation of entropy is tantamount with a loss of total pressure. A dimensionless total pressure loss coefficient can be defined as

$$
\zeta=\frac{p_{\text {tot,in }}-p_{\text {totout }}}{p_{\text {tot,in }}-p_{\text {in }}}
$$

It can be shown per the Bernoulli equation that $c_{p}$, $\xi$, and $\zeta$ are related via

$$
c_{p}+\xi+\zeta=1
$$

Equation (6) shows that within the pressure budget a decrease in total pressure loss results in a change of pressure recovery and kinetic energy coefficient. Two respective examples of this interdependence shall be given:

The pressure recovery primarily depends on the diffuser geometry, see Equation (2). Shorter diffuser designs are advantageous as the shorter flow paths result in less frictional total pressure losses. Additionally, shorter diffusers yield reduced investment costs. Given a constant area ratio, as the length of a diffuser decreases, its opening angle becomes steeper and the diffuser flow becomes more prone to boundary layer separation, which is a source for total pressure loss.

The residual kinetic energy is often considered as unexploited energy when focusing solely on pressure recovery, but in context of a power plant, kinetic energy is required to drive the flow through the exhaust stack downstream of the diffuser. This is especially important if the gas turbine is operated in a combined cycle where a heat recovery steam generator follows downstream of the diffuser. This type of component introduces a considerable flow resistance within the flow path, where lots of kinetic energy is dissipated. Therefore a certain amount of residual kinetic energy is essential for the overall process.

In conclusion, there is a strong interdependence between total pressure loss, pressure recovery and residual kinetic energy: an overall reduction of total pressure losses benefits the diffuser design, in particular if the diffuser is considered as a part of a highly integrated system, like power plants. We consider a combined approach towards the design and its adjacent components to be of cardinal importance. 


\section{Stabilisation number}

A basis for evaluating the pressure recovery of a diffuser design are empirical diffuser charts, e.g., Sovran and Klomp (1967) or ESDU (1990). However, these charts rely on simplified inflow conditions and require additional, semi-empirical corrections to account for non-uniform inflow conditions. For example, Vassiliev et al. (2011) showed that the diffuser performance depends on the inlet Mach number, total pressure distribution, flow angle, and turbulence characteristics.

In addition to the static inlet conditions Kluß et al. (2009) numerically investigated the effects of unsteady wakes and secondary flows shed from rotating cylindrical spokes upstream of the diffuser on the pressure recovery. In contrast to predictions made using diffuser design charts assuming static inlet conditions, the unsteady inflow caused a re-attachment of the boundary layer. This results in an increase in pressure recovery exceeding static predictions. The findings were experimentally validated by Sieker and Seume (2008). Kuschel and Seume (2011) conducted additional experiments using a NACA profile instead of cylindrical spokes. A detailed analysis of these experimental results can be found in Kuschel et al. (2015) and Drechsel et al. (2015). They conclude that the increase in pressure recovery is caused by the interaction of tip vortices of the rotor blades with the diffuser boundary layer. The origin of theses vortices in the rotor tip region is further investigated by Drechsel et al. (2016). All these findings show that a combined design methodology for the last turbine stage and the diffuser is beneficial in achieving more efficient designs.

The stabilising properties of tip leakage vortices generated in the last rotor row and their effect on the boundary layer characteristics have been examined in Mimic et al. (2018). A correlation between the pressure recovery of the diffuser and integral rotor parameters of the last stage has been established, based on analytical considerations, numerical simulations, and experimental data. Parts of the experimental data have previously been published in Kuschel (2014). These rotor parameters are loading coefficient $\Psi$, flow coefficient $\Phi$, and reduced frequency $f_{\text {red }}$ (see Equations A1 to A3, as detailed in Appendix A).

Both experimental data and scale-resolving simulations, carried out with the SST-SAS method, showed excellent agreement with the correlation. The three parameters have been further condensed into the stabilisation number $\Sigma$ which is defined as

$$
\Sigma \equiv \frac{\Psi f_{\text {red }}}{\Phi^{2}}
$$

The number represents a measure for the prediction of the diffuser effectiveness $\epsilon$. With the definition of the stabilisation number $\Sigma$ presented in Mimic et al. (2018), the correlation then gives

$$
\Delta \epsilon_{\text {corr }}(\Sigma) \approx 2.45 \Sigma
$$

as depicted in Figure 1. The correlation shows that operating points with higher values for the stabilisation number $\Sigma$-i.e., essentially higher blade loading, more circumferential trajectories of the blade tip vortices, and more vortex passings per unit time- exhibit an increased diffuser effectivity. A change in $\Sigma$ can be attributed to deliberate blade design choices regarding the last turbine stage as well as part-load operation for a given engine design.

\section{Total pressure loss coefficient}

The total pressure loss in diffusers can be attributed to several sources. From a design point of view the losses caused by struts within the flow path, especially with high incidence flow during part-load, and the losses in the Carnot diffuser between the annular and conical diffuser are of great interest. The great amount of research conducted makes this evident, see for example Hirschmann et al. (2012), Vassiliev et al. (2014), Schäfer et al. (2014) or Seume and Drechsel (2015). In this paper, however, we consider just the annular part of the diffuser without struts.

The scope of this paper comprises the total pressure losses produced in the shroud boundary layer of the annular diffuser downstream of the rotor. Babu et al. (2011) were able to demonstrate that rotor tip leakage, achieved by means of flow injection in the shroud region, allow to decrease total pressure loss in comparison to an idealised uniform inflow. In the investigation presented here, the tip jet is achieved by a rotor instead. Therefore, a trade-off can be expected between the losses introduced by the tip vortex and the loss reduction by 
the homogenisation of the boundary layer and its delayed separation. As flow separation can generally be linked to a decrease in pressure recovery and an increase in total pressure loss, we propose the following hypothesis:

The total pressure loss coefficient $\zeta$ decreases for increasing values of the stabilisation number $\Sigma$.

Note that because this investigation takes place immediately downstream of the rotor, additional mixing losses, that are introduced by the wakes of the rotor entering the diffuser domain, must be taken into account.

\section{Analytical considerations}

According to Denton (1993), losses in turbomachinery can be divided into three categories: profile losses, endwall losses and leakage losses. In this paper, leakage losses do not play any significant role, as the diffuser discussed is a rather simplified model. In order to give a better representation of the flow phenomena in the diffuser, the remaining loss mechanisms can be rearranged into:

1. boundary layer losses,

2. secondary flow losses, and

3. wake mixing losses.

The first two loss mechanisms work in conflict: the vortical structures incoming from the rotor blade tips do reduce boundary layer thickness, even preventing separation and, as such, cause a reduction of boundary-layer-related losses. However, at the same time, these vortical structures can be shown to increase the secondary flow losses. In order to present a proper budgeting of the two adverse influences, a closer look shall be taken at the underlying flow mechanisms.

The third loss mechanism — the mixing of wakes incoming from the turbine—is somewhat different: because the factors, i.e., width, deficit, and shape of the wakes that determine the mixing losses are strongly dependent on the rotor geometry, the exact interactions are beyond the scope of this paper. We do, however, propose a rather simple rectification of the wake-induced losses for the given configuration later in this composition.

\section{Boundary layer losses}

For an ideal gas, the energy equation of a flow can be given in the following form:

$$
\rho \frac{\partial h}{\partial t}+\rho c_{i} \frac{\partial h}{\partial x_{i}}=\frac{\partial}{\partial x_{i}}\left(\lambda \frac{\partial T}{\partial x_{i}}\right)+\frac{\partial p}{\partial t}+c_{i} \frac{\partial p}{\partial x_{i}}+\underbrace{2 \mu s_{i j} s_{i j}}_{\mathcal{D}_{e}}
$$

where $s_{i j}$ is defined as the traceless strain rate tensor, i.e.,

$$
s_{i j}=\left(\frac{1}{2}\left(\frac{\partial c_{i}}{\partial x_{j}}+\frac{\partial c_{j}}{\partial x_{i}}\right)-\frac{1}{3} \frac{\partial c_{k}}{\partial x_{k}} \delta_{i j}\right)
$$

The last term $\mathcal{D}_{e}$ on the right-hand side of Equation (9) describes the dissipation of kinetic energy as heat. Therefore, a reduction of the wall-normal velocity gradients reduces the dissipation losses in the boundary layer. As can be seen in Figure 2, this is exactly what happens to the boundary layer velocity profile of the diffuser, when the rotor outflow is characterised by a high stabilisation number $\Sigma$.

\section{Secondary flow losses}

While the increased diffuser effectiveness and reduced total pressure losses in the boundary layer are certainly favourable, the effect of additional secondary flow structures in the diffuser would benefit from further examination. From the dot product of the vorticity $\omega_{i}$ and the vorticity equation, i.e.,

$$
\rho \frac{\partial \omega_{i}}{\partial t}+\rho c_{j} \frac{\partial \omega_{i}}{\partial x_{j}}=\rho \omega_{j} \frac{\partial c_{i}}{\partial x_{j}}+\mu \frac{\partial^{2} \omega_{i}}{\partial x_{j} \partial x_{j}}
$$




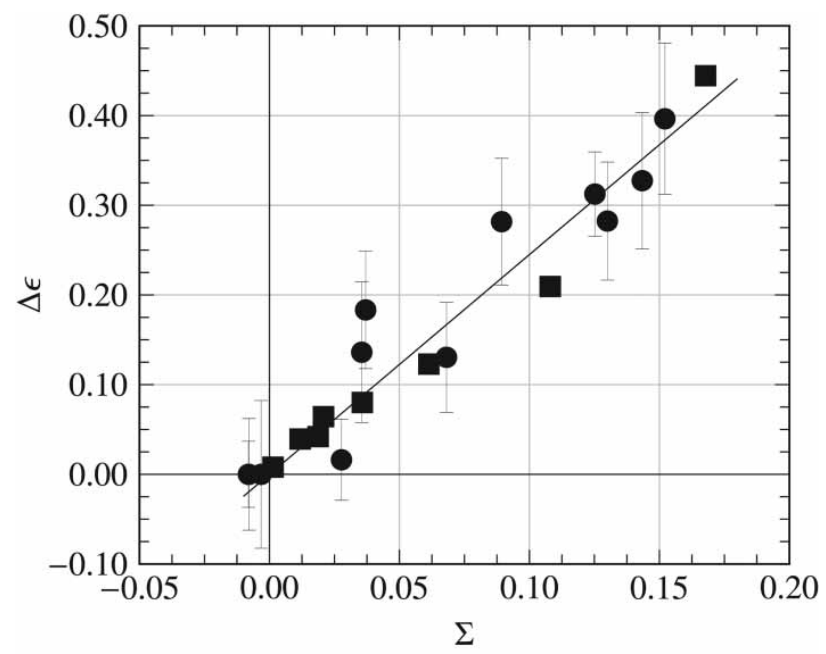

- Experiments

- Numerical simulations

Correlation: $\Delta \epsilon_{\text {corr }}(\Sigma) \approx 2.45 \Sigma$

Figure 1. Increase in diffuser effectiveness from reference, $\Delta \epsilon$, against stabilisation number $\Sigma$ (Reproduced from Mimic et al., 2018).

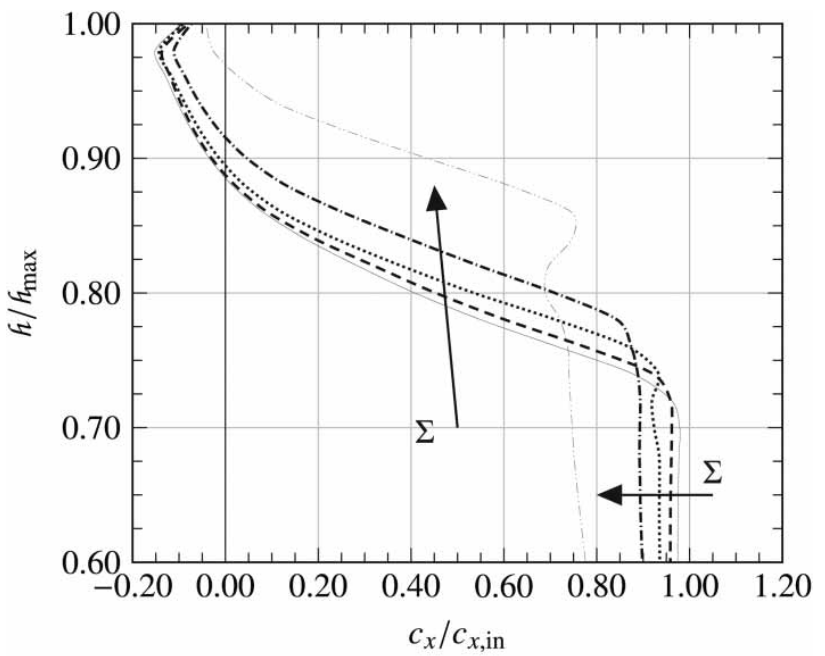

$\Sigma=0.0187-\cdots \quad \Sigma=0.0357$

$\Sigma=0.0613$

$\Sigma=0.1678$

Figure 2. Mass-flow-weighted, circumferentially averaged radial profile of non-dimensional axial velocity at $50 \%$ of diffuser length (Reproduced from Mimic et al., 2018).

follows the enstrophy equation, namely

$$
\rho \frac{\partial \omega_{i} \omega_{i}}{\partial t}+\rho c_{j} \frac{\partial \omega_{i} \omega_{i}}{\partial x_{j}}=\rho \omega_{i} \omega_{j} \frac{\partial c_{i}}{\partial x_{j}}+\mu \frac{\partial^{2} \omega_{i} \omega_{i}}{\partial x_{j} \partial x_{j}}-\underbrace{2 \mu \frac{\partial \omega_{i}}{\partial x_{j}} \frac{\partial \omega_{i}}{\partial x_{j}}}_{\mathcal{D}_{\mathcal{E}}}
$$

with the enstrophy $\mathcal{E}$ being defined as

$$
\mathcal{E}=\omega_{i}^{2}
$$

and giving a measure for the rotational kinetic energy of the flow field. Here, the last term on the right-hand side of Equation (12), $\mathcal{D}_{\mathcal{E}}$, represents enstrophy dissipation and is strictly non-negative. Thus, in the presence of high vorticity gradients - as they are typically found in the perturbations caused by tip leakage flow-enstrophy is dissipated as heat. This entails increased vortex-induced losses for operating points with high values of $\Sigma$.

Note, however, that dissipation of energy and dissipation of enstrophy should not be understood as two summands of some kind of total, or combined, dissipation. The same as enstrophy describes a filtered feature of the velocity field, namely its rotation, the dissipation of enstrophy gives a measure for the portion of energy dissipation that is caused by said rotation.

Because the interactions between the boundary layer in the diffuser and the blade tip vortices are complex and not necessarily linear-meaning that they cannot simply be superimposed onto each other-a mere analytical prediction would be inaccurate, at best. Therefore, we present experimental data and numerical simulations to evaluate the extent of the individual influence factors and to assess their effect on overall total pressure loss production.

\section{Test facility}

Experimental investigations were carried out on the low-speed axial diffuser test rig at the Institute of Turbomachinery and Fluid Dynamics. The test rig represents a heavy-duty exhaust diffuser at a scale of 1:10. The diffuser is divided into an annular and a conical section, as shown in Figure 3. The half-opening angle $\delta_{\mathrm{AD}}$ of the annular section is $15^{\circ}$. The boundary layer of the annular diffuser is susceptible to separation for steady homogeneous inflow conditions, according to the diffuser charts of Sovran and Klomp (1967). A rotating wake generator provides inflow conditions for the diffuser, similar to the conditions at the outlet of a low-pressure 


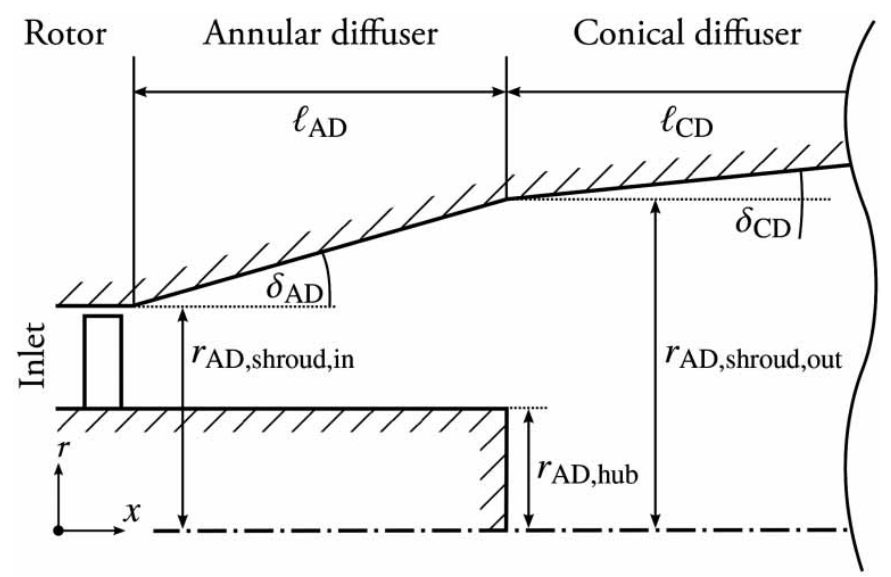

Figure 3. The diffuser test rig at the Institute of Turbomachinery and Fluid Dynamics represents a 1:10 scaled heavy-duty exhaust diffuser with an annular and a conical diffuser part.

turbine. Two interchangeable rotating wake generators are used, that consist of 30 and 15 symmetric NACA0020 blades, respectively. The aerodynamic blade loading equals zero at design operating conditions. Additional test rig parameters can be found in Table 1.

\section{Numerical method}

All presented simulations were carried out using the non-commercial solver TRACE 8.2 (Turbomachinery Research Aerodynamics Computational Environment). TRACE is developed by the Institute of Propulsion Technology at the German Aerospace Center (DLR). Turbulence is modelled using SST-SAS and fully turbulent boundary layer treatment at the walls. SST-SAS describes the combination of the Scale Adaptive Simulation (SAS) method by Menter and Egorov (2010), and the k- $\omega$-SST turbulence model by Menter (1994) and is used to facilitate the formation of unsteady flow structures such as boundary layer separations and vortices. A stagnation point anomaly fix according to Kato and Launder (1993) is employed. A blending function from Strelets (2001) is used, in order to switch between a second order central differencing scheme in SAS-dominated flow regions and a second order upwind differencing scheme in RANS-dominated flow regions. The object is to reduce numerical dissipation in the former and to enhance stability in the latter. Further details concerning the numerical setup are explained in Mimic et al. (2018).

\section{Computational domain}

The numerical domain represents one pitch of the rotor. The numerical simulations feature-different than in the experiments_-blade counts of 25, 30 and 40. The blade has a symmetric NACA0020 profile and is unloaded at the aerodynamic design point. The rotor domain is followed by an annular diffuser with a half-opening angle

Table 1. Geometric properties of the test rig.

\begin{tabular}{|l|c|l|c|}
\hline \multicolumn{2}{|l|}{ Rotor properties } & \multicolumn{2}{c|}{ Diffuser properties } \\
\hline Shape & NACA0020 & $\ell_{\mathrm{AD}}$ & $235 \mathrm{~mm}$ \\
\hline Blade count & $15 / 30$ & $\ell_{\mathrm{CD}}$ & $1,735 \mathrm{~mm}$ \\
\hline Hub radius & $140 \mathrm{~mm}$ & $r_{\mathrm{AD}, \text { hub }}$ & $140 \mathrm{~mm}$ \\
\hline Blade height & $97 \mathrm{~mm}$ & $r_{\mathrm{AD}, \text { shroud,in }}$ & $238 \mathrm{~mm}$ \\
\hline Tip clearance & $1 \mathrm{~mm}$ & $r_{\mathrm{AD}, \text { shroud,out }}$ & $300 \mathrm{~mm}$ \\
\hline Stagger angle at hub/tip & $43^{\circ} / 58^{\circ}$ & $\delta_{\mathrm{AD}} / \delta_{\mathrm{CD}}$ & $15^{\circ} / 5^{\circ}$ \\
\hline
\end{tabular}




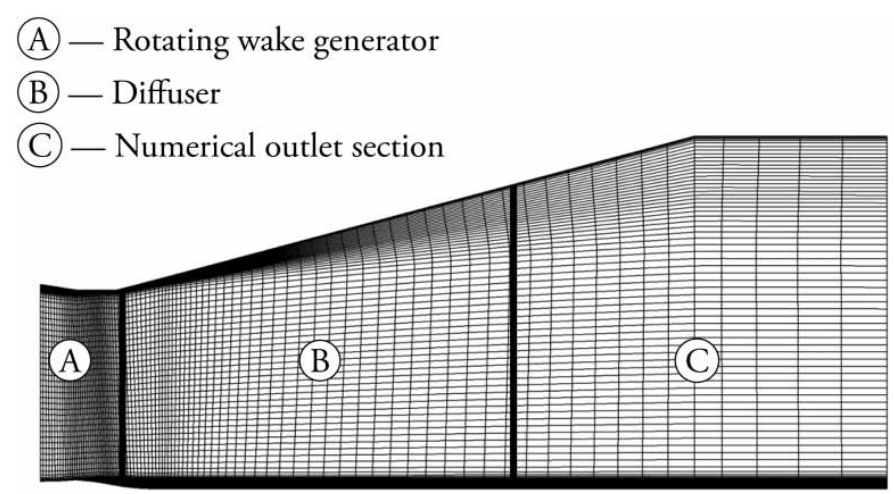

Figure 4. Computational Domain (coarse mesh for display): The entire domain is simulated inside the rotating system (Reproduced from Mimic et al., 2018).

of $15^{\circ}$ leading to a numerical outlet section that is made up of a divergent and a straight duct. The entire numerical domain, as shown in Figure 4, is in a rotating frame of reference. The numerical reference planes for the evaluation of the overall diffuser total pressure loss coefficient $\zeta$ match the experiment. They are located $15 \mathrm{~mm}$ downstream of the diffuser inlet and at diffuser outlet.

The mesh consists of 1.7-2.4 million overall cells, depending on the blade count of the respective mesh. The mesh is refined in and around the tip gap as well as in the diffuser shroud region. Here, unsteady effects are to be expected due to vortex generation and massive boundary layer separation. A numerical outlet section-downstream of the nominal diffuser outlet—coarsened in axial direction leads to locally elevated numerical dissipation. This way, eventual disturbances interacting with the outlet boundary condition are damped. Numerical convergence and stability are enhanced, as a result. Because the whole numerical domain is part of the rotating relative system, no interface is needed between rotor and diffuser. Static pressure values are given as outlet boundary conditions. The outlet pressure is adjusted to match the required mass flow rate for each individual operating point. An extensive grid convergence study with a similar grid and the SST model has been carried out by Drechsel et al. (2015).

\section{Analysis}

We present a range of operating points differing from each other in $\Psi, \Phi$, and $f_{\text {red }}$ by varying the blade count $n$, rotor speed $N$, and mass flow rate $\dot{m}$. The test cases comprise numerical (NUM) and experimental (EXP) test cases, which are listed in Table 2. The test cases have been presented in Mimic et al. (2018). We averaged between identical operating points of EXP that were measured multiple times.

Samples № 3, 6, and 11 are essentially incidence-free and exhibit no turning. The negative values listed for № 3 and 6 are most probably due to measurement inaccuracies, which are negligible. Hence, variants № 3 and 6 are used as the respective references for the experiments; sample 11 represents the reference for numerical simulations. For all cases examined, the flow coefficient is equal to or greater than the design flow coefficient at reference conditions, resulting in a change in incidence. The work coefficient is mostly greater than zero, as this would be expected for a turbine.

\section{Rectified total pressure loss}

The absolute rotor outflow angle $\alpha$ depends on the operating point. Thus, the fluid travels a longer distance in the diffuser with regards to a swirl-free rotor outflow. Trigonometry shows that the length of a streamline is inversely proportional to $\cos \alpha$. Because total pressure losses are expected to increase with the distance covered, the total pressure loss coefficients for the respective operating points are rectified to an equivalent, swirl-free pressure loss coefficient.

Because the definition of the stabilisation number $\Sigma$ is based on the behaviour of the tip leakage vortices, it cannot account for the effect of wakes generated by the rotor. A simple numerical analysis reveals that the wakes become more accentuated for higher flow coefficients $\Phi$ - this equals a higher incidence of the rotor inflowwhich manifests in greater wake velocity deficits as shown in Figure 5. As can be seen, some oscillations are present in the free-stream region. Even though they bear no significance on the overall result, this is a typical 
Table 2. Test cases (Mimic et al., 2018).

\begin{tabular}{|c|c|c|c|c|c|c|c|c|}
\hline № & & $n$ & $\begin{array}{c}N \\
\text { in } \mathrm{RPM}\end{array}$ & $\begin{array}{c}\dot{m} \\
\text { in } \mathrm{kg} / \mathrm{s}\end{array}$ & $\Psi$ & $\Phi$ & $f_{\text {red }}$ & $\Sigma$ \\
\hline 1 & \multirow{8}{*}{ EXP } & 15 & 1,500 & 4.8 & 0.19 & 1.10 & 0.22 & 0.0355 \\
\hline 2 & & 15 & 1,500 & 5.3 & 0.27 & 1.21 & 0.20 & 0.0370 \\
\hline 3 & & 15 & 2,500 & 5.3 & -0.01 & 0.73 & 0.34 & -0.0079 \\
\hline 4 & & 15 & 2,500 & 6.3 & 0.07 & 0.86 & 0.28 & 0.0277 \\
\hline 5 & & 30 & 1,500 & 4.8 & 0.33 & 1.08 & 0.46 & 0.1300 \\
\hline 6 & & 30 & 2,500 & 5.3 & -0.00 & 0.74 & 0.66 & -0.0032 \\
\hline 7 & & 30 & 1,500 & 5.4 & 0.45 & 1.21 & 0.41 & 0.1252 \\
\hline 8 & & 30 & 2,500 & 6.3 & 0.09 & 0.85 & 0.58 & 0.0681 \\
\hline 9 & \multirow{8}{*}{ NUM } & 25 & 1,875 & 5.5 & 0.20 & 1.10 & 0.37 & 0.0613 \\
\hline 10 & & 25 & 2,500 & 5.5 & 0.03 & 0.82 & 0.50 & 0.0187 \\
\hline 11 & & 30 & 2,500 & 5.1 & 0.00 & 0.78 & 0.63 & 0.0014 \\
\hline 12 & & 30 & 2,500 & 5.2 & 0.01 & 0.79 & 0.62 & 0.0118 \\
\hline 13 & & 30 & 2,500 & 5.3 & 0.02 & 0.81 & 0.61 & 0.0208 \\
\hline 14 & & 30 & 2,500 & 5.5 & 0.04 & 0.83 & 0.59 & 0.0357 \\
\hline 15 & & 40 & 1,875 & 5.5 & 0.38 & 1.14 & 0.58 & 0.1678 \\
\hline 16 & & 40 & 2,500 & 5.5 & 0.10 & 0.85 & 0.77 & 0.1080 \\
\hline
\end{tabular}

issue that arises when RANS computations are performed using central differencing schemes. It is caused by inaccuracies in the performance of the blending function from Strelets (2001).

Additionally it is assumed that the pressure loss coefficients associated with wake mixing are roughly proportional to the square of the absolute rotor outflow velocity $c_{\mathrm{II}}^{2}$ for the parameter range discussed in this paper. For the above reasons, we propose a rectified total pressure loss coefficient $\varpi$ which is defined as

$$
\varpi=\left(\frac{\Upsilon_{\text {rel }}}{c_{\mathrm{II}, \mathrm{rel}}^{2} \Phi_{\mathrm{rel}}}\right) \zeta
$$

where $\Upsilon_{\text {rel }}, \Phi_{\text {rel }}$ and $c_{\text {II,rel }}^{2}$ are defined as

$$
\Upsilon_{\text {rel }} \equiv \frac{\cos \alpha}{\cos \alpha_{\text {ref }}}, \Phi_{\text {rel }}=\frac{\Phi}{\Phi_{\text {ref }}}, \quad \text { and } \quad c_{\text {II, rel }}=\frac{c_{\text {II }}}{c_{\text {II, ref }}}
$$

Note that the relative rotor outflow velocity $c_{\mathrm{II}, \text { rel }}$ is still in the absolute frame of reference and must not be confused with the rotor outflow velocity in a relative frame of reference.

Of course, the assessment of wake mixing losses can be done in greater detail, which is, however, beyond the scope of this paper. Examples for such calculations are given by Denton (1993) as well as Rose and Harvey (1999). 


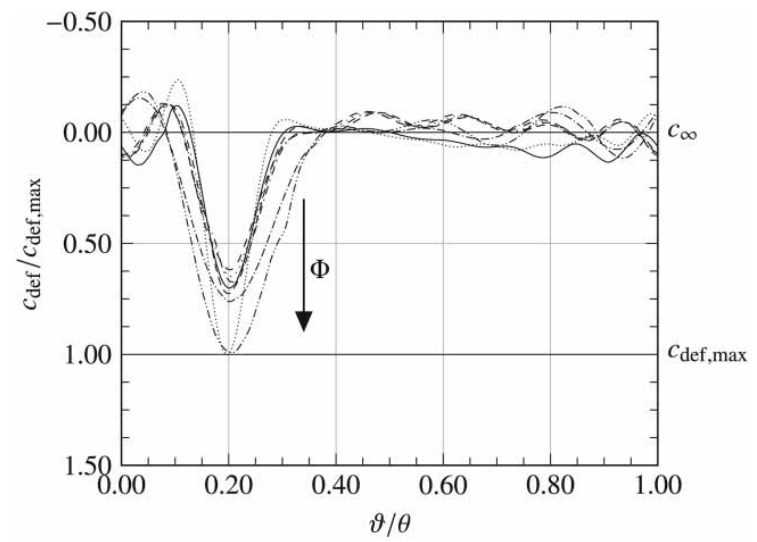

$$
\begin{aligned}
& \text { — } \Sigma=0.0187 \ldots \Sigma=0.0014 \\
& \Sigma=0.0118 \ldots \Sigma=0.0208 \\
& \ldots \quad \Sigma=0.0357 \quad \Sigma=0.0613 \\
& \Sigma=0.1080 \ldots \Sigma=0.1678
\end{aligned}
$$

Figure 5. Time-averaged circumferential distribution of non-dimensional wake velocity deficit at Euler radius at $8 \%$ of diffuser length.

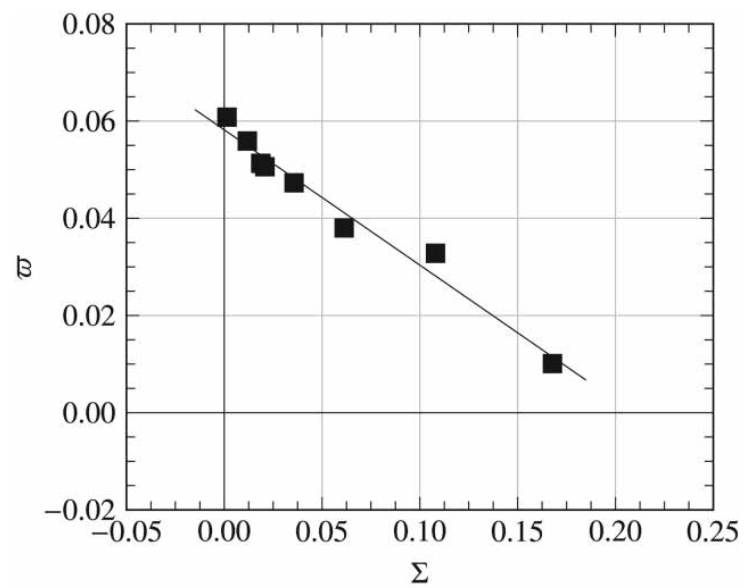

- Numerical simulations Corr.: $\varpi_{\text {corr,NUM }}(\Sigma) \approx-0.28 \Sigma+0.06$

Figure 6. Rectified diffuser total pressure loss $\varpi$ against stabilisation number $\Sigma$.

\section{The correlation}

Figure 6 shows for the numerical simulations performed that the rectified diffuser pressure loss coefficient $\varpi$ decreases in a linear way for increasing values of the stabilisation number $\Sigma$. The following correlation can be given from the numerical samples:

$$
\varpi_{\text {corr,NUM }}(\Sigma)=-0.2784 \Sigma+0.0582 \text { with } R^{2}=0.9724
$$

It is, however, physically impossible for $\varpi$ to attain negative values. Anyhow, for excessively increasing values of $\Sigma$, one may expect drastically increasing secondary flow losses.

The absolute difference in rectified total pressure loss coefficient to the respective reference variants for simulations and experiments,

$$
\Delta \varpi=\varpi-\varpi_{\text {ref }}
$$

facilitates the comparison between record sets. The result is shown in Figure 7. Again, a linear correlation for both, experiment and numerical simulations, between $\Delta \varpi$ and $\Sigma$ becomes apparent-here with a slightly steeper descent than just for the numerical results. The correlation gives

$$
\Delta \varpi_{\mathrm{corr}}(\Sigma)=-0.3842 \Sigma-0.0016 \quad \text { with } R^{2}=0.8136
$$

or, for a regression that goes through the point of origin

$$
\Delta \varpi_{\mathrm{corr}}(\Sigma) \approx-0.4 \Sigma \quad \text { with } R^{2} \approx 0.81
$$

Since $\varpi_{\text {ref }}$ equals $\zeta_{\text {ref, }}$ the correlation can be simplified to

$$
\zeta_{\text {corr }}(\Lambda, \Sigma) \approx \Lambda-0.4 \Sigma+\zeta_{\text {ref }}
$$

Here, we introduce the loss rectification factor $\Lambda$ to quantify the effect of wakes and other non-vortex-induced phenomena. For the rotor investigated, it is defined as

$$
\Lambda \equiv \frac{c_{\text {II,rel }}^{2} \Phi_{\text {rel }}}{\Upsilon_{\text {rel }}}
$$



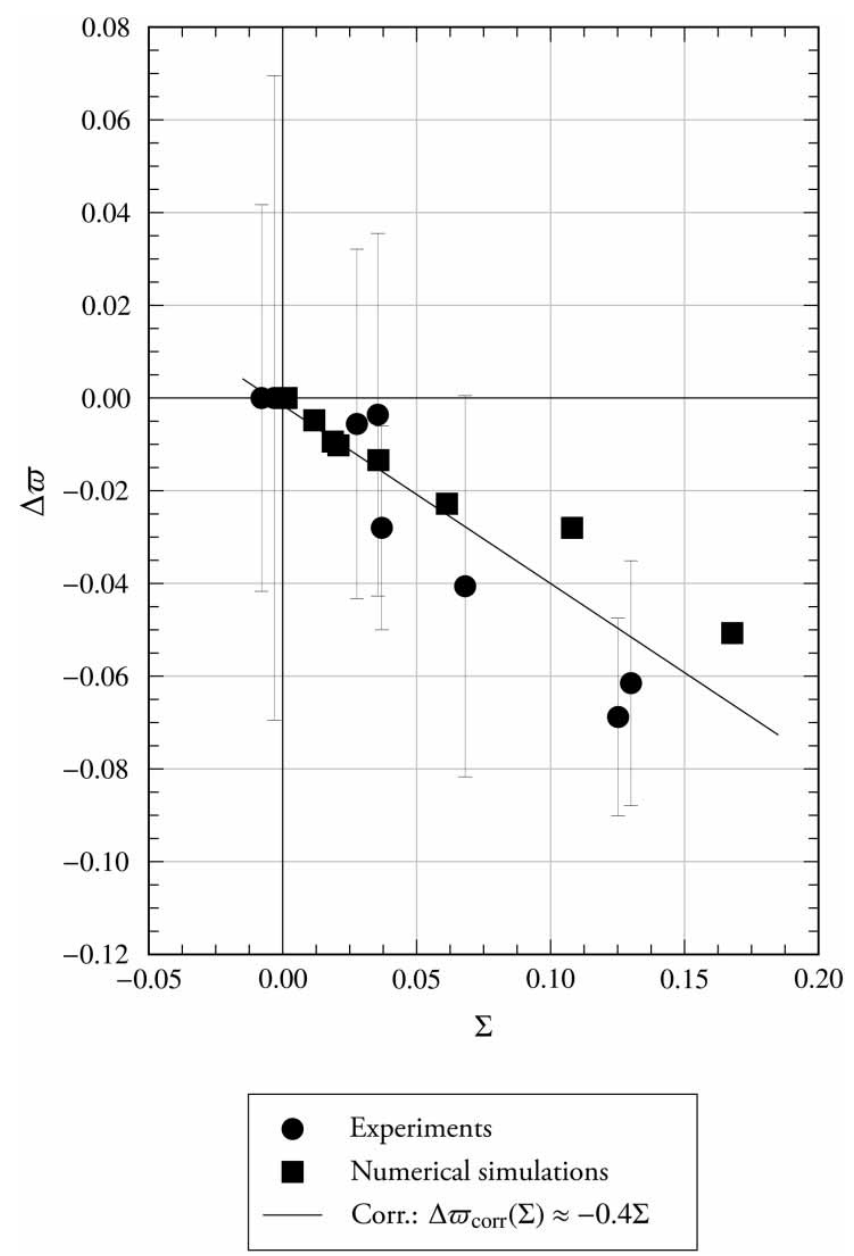

Figure 7. Absolute difference in rectified diffuser total pressure loss from reference, $\Delta \varpi$, against stabilization number $\Sigma$.

We suspect that the exact definition of $\Lambda$ is dependent on the exact geometry of the rotor and may require additional rectification factors. Regardless, this requires further studies. While the correlation proposed in Equation (20) indicates a clear relation between total pressure losses generated in the diffuser and the degree of boundary layer stabilisation its physical underpinnings shall be further discussed.

\section{Dissipation}

As initially discussed, the dissipation terms of the energy equation and the enstrophy equation, $\mathcal{D}_{e}$ and $\mathcal{D}_{\mathcal{E}}$, respectively, indicate loss production. While the former gives a measure for the entirety of losses produced, the latter allows to detect the losses caused by vortical structures. An analysis of the respective distributions of both terms in the numerical solutions allows to better understand where irreversible flow processes lead to total pressure drops.

Unlike the term given for $\mathcal{D}_{e}$ in Equation (9), the turbulent energy dissipation,

$$
\mathcal{D}_{e, t} \equiv 2\left(\mu+\mu_{t}\right) s_{i j} s_{i j}
$$

shall be used in the following. In this formulation, the viscosity $\mu$ has been replaced by the sum of molecular and turbulent viscosity $\mu+\mu_{t}$ to account for the influence of turbulence. Figure 8 shows the circumferentially averaged relative magnitude of $\mathcal{D}_{e, t}$ in the shroud near region for different operating points.

Apparently, the dissipation of energy decreases in intensity and expanse for increasing diffuser stability. This is consistent with the homogenisation of the velocity profiles seen in Figure 2. The five variants with the lowest stabilisation numbers (from bottom to top in Figure 8) - which are also located close to each other in Figure 6are fairly similar, whereas a considerable weakening of the dissipation can be observed from $\Sigma=0.0613$ to $\Sigma=0.1678$. 


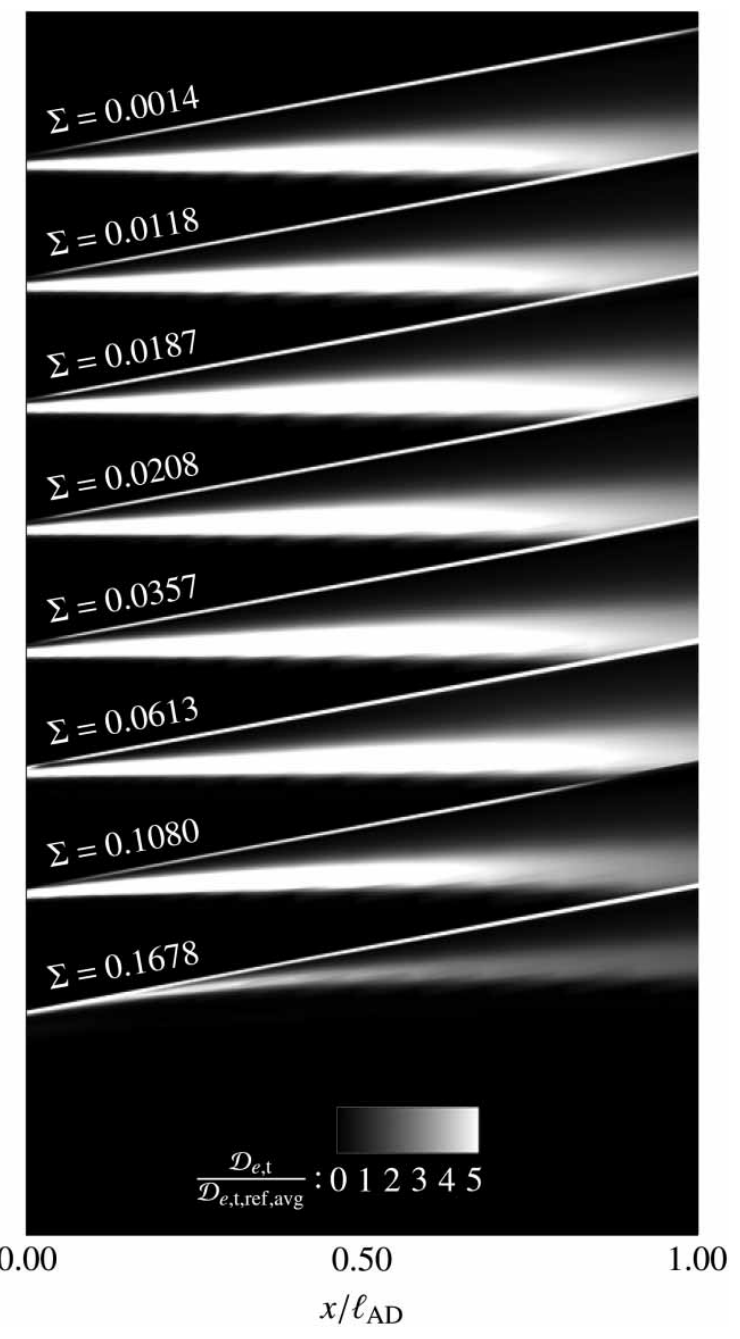

Figure 8. Circumferentially averaged distribution of non-dimensional energy dissipation $\mathcal{D}_{\mathrm{e}, \mathrm{t}} / \mathcal{D}_{\mathrm{e}, \mathrm{t}, \mathrm{ref}, \mathrm{avg}}$ for different values of $\Sigma$.

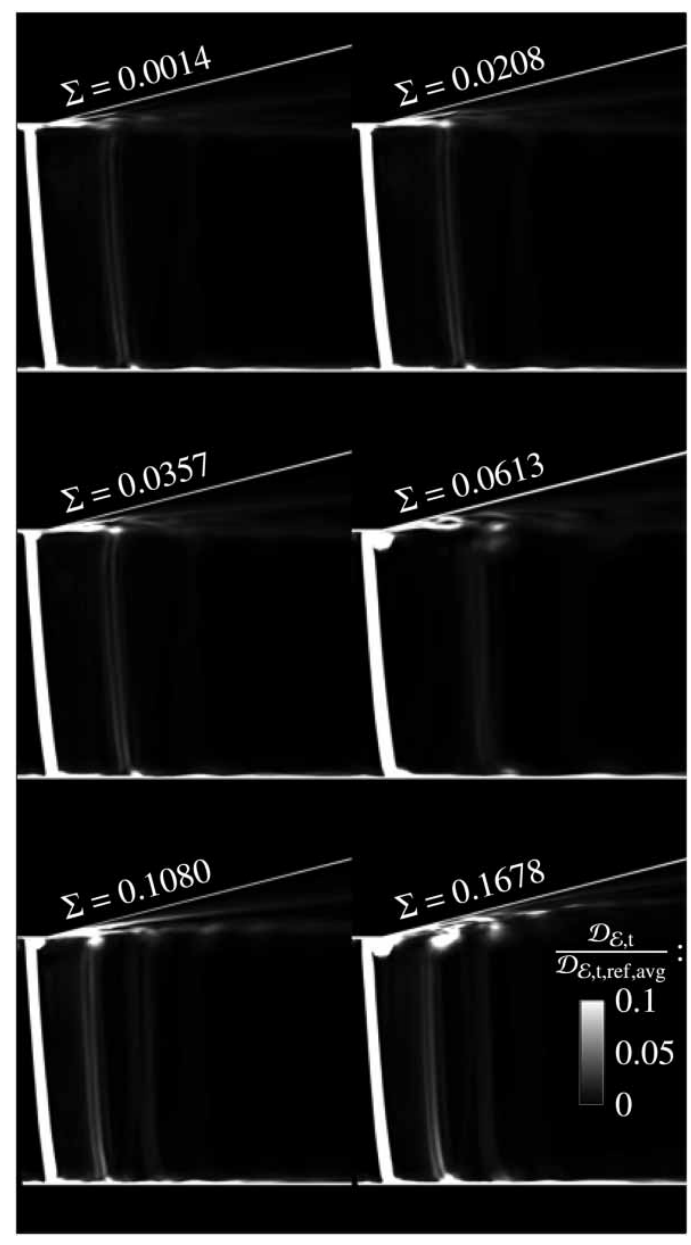

Figure 9. Meridional plane showing the time averaged distribution of non-dimensional enstrophy dissipation

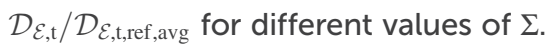

Conversely, Figure 9 depicts the turbulent dissipation of enstrophy for different, representative operating points, that is,

$$
\mathcal{D}_{\mathcal{E}, t} \equiv 2(\mu+\mu) \frac{\partial \omega_{i}}{\partial x_{j}} \frac{\partial \omega_{i}}{\partial x_{j}}
$$

The contour plots are taken for meridional planes, which are oriented so that the trailing edge of the blade lies directly behind the plane. The slanted, white bar at the left of the individual figure frames is caused by the wakes and indicates the position of the trailing edge. One can see that the highly vortical tip leakage flow causes considerable enstrophy dissipation. An effect that becomes more accentuated for increasing values of $\Sigma$, as the tip leakage vortex becomes more powerful.

While the magnitudes of $\mathcal{D}_{e, t}$ and $\mathcal{D}_{\mathcal{E}, t}$ cannot be adequately compared to each other, the vastly different spatial dimensions make it clear that the effect of vortex-induced losses is more locally limited and almost vanishes in comparison to the dominating boundary layer and separation losses for the diffuser rig discussed.

\section{Conclusions}

The total pressure loss coefficient of the annular diffuser tends to decrease for growing stabilisation numbers. This is due to a more homogeneous radial flow profile stemming from pronounced interactions between the blade tip vortices and the boundary layer. The total pressure loss in the diffuser correlates well with the stabilisation number $\Sigma$, especially if wake mixing and swirl are accounted for in the form of a rectified total pressure loss 
coefficient $\varpi$. We derive a correlation for the total pressure loss coefficient $\zeta$ from a wide range of numerical simulations and experimental results. The correlation can be broken down into two parts. The first part is independent from the rotor geometry, whereas the second part is suspected to be rotor-dependent. The latter is condensed into the loss rectification number $\Lambda$.

Therefore, we conclude that the results support the hypothesis initially stated-namely that the total pressure loss coefficient $\zeta$ decreases for increasing values of the stabilisation number $\Sigma$-if wake mixing and swirl-induced losses are accounted for.

We show that the loss mitigation is a consequence of the homogenisation of the boundary layer profile. This leads to smaller strain rates, which, in turn, reduces energy dissipation in the shroud near region of the diffuser. Even though dissipation of enstrophy contained in the wakes (which are accounted for, anyway) and the tip leakage vortices rises for growing values of $\Sigma$, this effect is locally confined and loses relative significance when unstable boundary layers and flow separations are present. Future research should emphasise further generalisation of the loss correlation presented. This includes studies on different diffuser opening angles, turbine-specific blade profiles, variations of the tip gap as well as DDES or LES of the influence of transition and flow separation happening on the blades or shocks in transonic low-pressure turbines.

\section{Nomenclature}

Unless otherwise noted only SI units are used.

\section{Symbols}

$\mathrm{AR}$ area ratio of the diffuser

$c_{i} \quad$ flow velocity

$c_{p} \quad$ pressure recovery coefficient

$\mathcal{D}$ dissipation

$e$ energy

$\varepsilon \quad$ enstrophy

$f_{\text {bp }} \quad$ blade passing frequency

$f_{\text {red }}$ reduced frequency

$h$ enthalpy (default: static)

b height-wise coordinate

$\ell \quad$ length

$\ell_{c} \quad$ chord length

$\dot{m}$ mass flow rate

$n \quad$ blade count

$N \quad$ rotational speed in revolutions per minute

$p \quad$ pressure (default: static)

$r, r$ radius, Euler radius

$R^{2} \quad$ coefficient of determination

$s_{i j} \quad$ traceless strain tensor

$t \quad$ time

$T$ temperature (default: static)

$u$ rotational velocity

$x_{i} \quad$ generalised spatial coordinate

$x \quad$ axial coordinate

$\alpha \quad$ flow angle, swirl angle

$\delta$ diffuser half-opening angle

$\epsilon \quad$ diffuser effectiveness

$\zeta \quad$ total pressure loss coefficient

$\vartheta \quad$ circumferential coordinate

$\theta \quad$ single pitch

$\lambda$ thermal conductivity

$\Lambda \quad$ loss rectification number

$\mu \quad$ dynamic viscosity

$\mu_{t} \quad$ turbulent eddy viscosity

$\xi \quad$ kinetic energy coefficient 
$\varpi \quad$ rectified total pressure loss coefficient

$\rho$ density

$\Sigma \quad$ stabilisation number

$\Phi, \Psi$ flow coefficient, loading coefficient

$\omega \quad$ vorticity

\section{Subscripts}

I, II rotor inlet plane, rotor exit plane

$\mathrm{AD}, \mathrm{CD}$ annular diffuser, conical diffuser

avg average over domain

def deficit

corr correlated

dyn dynamic quantity

in, out diffuser inlet/outlet

ref reference

rel relative

t turbulent quantity

tot total quantity

$i, j \quad$ generic indices

$\infty$ free-stream quantity

\section{Appendix A: Definition of the Rotor Design Parameters}

The rotor design parameters are defined as follows (Wilson and Korakianitis, 2014):

$$
\begin{gathered}
\Psi \equiv \frac{c_{\vartheta, \mathrm{I}}-c_{\vartheta, \mathrm{II}}}{u} \\
\Phi \equiv \frac{c_{x}}{u} \\
f_{\text {red }} \equiv \frac{n \frac{N}{60} \ell_{c}}{c_{x}}
\end{gathered}
$$

\section{Supporting material}

SI1. Data from Table 2 as tab-separated ASCII file. (TAB)

SI2. Data from Figure 1 as tab-separated ASCII file. (TAB)

SI3. Data from Figure 6 as tab-separated ASCII file. (TAB)

SI4. Data from Figure 7 as tab-separated ASCII file. (TAB)

\section{Acknowledgements}

We would like to acknowledge the valuable contribution of the DLR Institute of Propulsion Technology and MTU Aero Engines AG for providing TRACE and appreciate the support from Martin Franke (DLR) for implementing the SST-SAS turbulence model into TRACE. We also thank our former colleague Bastian Drechsel for providing experimental data for this paper.

\section{Funding sources}

None.

\section{Competing interests}

Dajan Mimic declares that he has no conflict of interest. Christoph Jätz declares that he has no conflict of interest. Florian Herbst declares that he has no conflict of interest. 


\section{References}

Babu M., Bhatia D., Shukla R. K., Pradeep A., and Roy B. (2011). Effect of turbine tip leakage flows on exhaust diffuser performance. Proc. ASME 54679, Vol. 7, 609-618. https://doi.org/10.1115/GT2011-45457

Denton J. D. (1993). Loss mechanisms in turbomachines. ASME Journal of Turbomachinery. 115 (4): 621-656. https://doi.org/10. $1115 / 1.2929299$

Drechsel B., Müller C., Herbst F., and Seume J. R. (2015). Influence of turbulent flow characteristics and coherent vortices on the pressure recovery of annular diffusers part B: scale-resolving simulations. Proc. ASME 56635, Vol. 2A, V02AT38A010. https://doi.org/10. 1115/GT2015-42477

Drechsel B., Seume J. R., and Herbst F. (2016). On the numerical prediction of the influence of tip flow on diffuser stability. International Journal of Gas Turbine, Propulsion and Power Systems (JGPP). 8 (3): V08N03TP04.

ESDU (1990). Introduction to Design and Performance Data for Diffusers. Data Item Number 76027, ISBN-13: 978-0856791642

Hirschmann A., Volkmer S., Casey M., and Montgomery M. (2012). Hub extension in an axial gas turbine diffuser. Proc. ASME 44748, Vol. 8, 1229-1238. https://doi.org/10.1115/GT2012-68832

Kato M. and Launder B. E. (1993). The modeling of turbulent flow around stationary and vibrating square cylinders. 9th Symposium on Turbulent Shear Flows, 10.4.1-10.4.6.

Kluß D., Stoff H., and Wiedermann A. (2009). Effect of wakes and secondary flow on re-attachment of turbine exit annular diffuser flow. ASME Journal of Turbomachinery. 131 (4): 041012. https://doi.org/10.1115/1.3070577

Kuschel M. (2014). Einfluss von Sekundärströmungen auf den Druckrückgewinn in Axialdiffusoren. PhD thesis, Leibniz Universität Hannover, Germany.

Kuschel M. and Seume J. R. (2011). Influence of unsteady turbine flow on the performance of an exhaust diffuser. Proc. ASME 54679, Vol. 7, 1551-1561. https://doi.org/10.1115/GT2011-45673

Kuschel M., Drechsel B., Kluß D., and Seume J. R. (2015). Influence of turbulent flow characteristics and coherent vortices on the pressure recovery of annular diffusers part A: experimental results. Proc. ASME 56635, Vol. 2A, V02AT38A009. https://doi.org/10.1115/ GT2015-42476

Menter F. R. (1994). Two-equation eddy-viscosity turbulence models for engineering applications. AIAA Journal of Fluids Engineering. 32 (8): 1598-1605. https://doi.org/10.2514/3.12149

Menter F. and Egorov Y. (2010). The scale-adaptive simulation method for unsteady turbulent flow predictions. Part 1: theory and model description. Flow, Turbulence and Combustion. 85 (1): 113-138. https://doi.org/10.1007/s10494-010-9264-5

Mimic D., Drechsel B., and Herbst F. (2018). Correlation between pressure recovery of highly loaded annular diffusers and integral stage design parameters. ASME Journal of Turbomachinery. 144 (7): 071002. https://doi.org/10.1115/1.4039821, under license CC-BY 4.0 .

Rose M. G. and Harvey N. W. (1999). Turbomachinery wakes: differential work and mixing losses. ASME Journal of Turbomachinery. 122 (1): 68-77. https://doi.org/10.1115/1.555429

Schäfer P., Gieß P., Finzel C., and Hofmann W. (2014). Some aspects on inlet blockage affecting the performance of a heavy duty gas turbine's exhaust diffuser. Proc. ASME. 45622, Vol. 2C, V02CT38A016. https://doi.org/10.1115/GT2014-25599

Seume J. R. and Drechsel B. (2015). Einfluss von Stützrippen auf die Sekundärströmung in Turbinendiffusoren. Final Report, Leibniz Universität Hannover, Institute of Turbomachinery and Fluid Dynamics. https://doi.org/10.2314/GBV:863128203

Sieker O. and Seume J. R. (2008). Influence of rotating wakes on separation in turbine exhaust diffusers. Journal of Thermal Science. 17 (1): 42-49. https://doi.org/10.1007/s11630-008-0042-9

Sovran G. and Klomp D. (1967). Experimentally determined optimum geometries for rectilinear diffusers with rectangular conical or annular cross-section. In: Fluid mechanics of internal flow, 270-319.

Strelets M. (2001). Detached eddy simulation of massively separated flows. 39th AIAA Aerospace Sciences Meeting and Exhibit, Aerospace Sciences Meetings. https://doi.org/10.2514/6.2001-879

Vassiliev V., Irmisch S., Abdel-Wahab S., and Granovskiy A. (2011). Impact of the inflow conditions on the heavy-duty gas turbine exhaust diffuser performance. ASME Journal of Turbomachinery. 134 (4): 041018. https://doi.org/10.1115/1.4003714

Vassiliev V., Sommer P., Granovsky A., and Prozorov S. (2014). Exhaust diffuser characteristics at off-design conditions. Proc. ASME. 45622, Vol. 2C, V02CT38A026. https://doi.org/10.1115/GT2014-25938

Wilson D. G. and Korakianitis T. (2014). The Design of High-Efficiency Turbomachinery and Gas Turbines, Second Edition. The MIT Press, Cambridge, MA. ISBN-13: 978-0262526685 